\title{
Editorial
}

\section{Self-Assembly of Semiconductor Metal Oxide Nanostructures}

\author{
Xiang Wu, ${ }^{1}$ Xijin Xu, ${ }^{2}$ Haibo Zeng, ${ }^{3}$ and Chuanfei Guo ${ }^{4}$ \\ ${ }^{1}$ Key Laboratory for Photonic and Electronic Bandgap Materials, Ministry of Education and College of Chemistry and \\ Chemical Engineering, Harbin Normal University, Harbin 150025, China \\ ${ }^{2}$ School of Physics and Technology, University of Jinan, Shandong, Jinan 250022, China \\ ${ }^{3}$ School of Materials Science and Engineering, Nanjing University of Science and Technology, \\ Nanjing 210094, China \\ ${ }^{4}$ Department of Physics, Boston College, Chestnut Hill, MA 02467, USA
}

Correspondence should be addressed to Xiang Wu; wuxiang05@gmail.com

Received 12 June 2013; Accepted 12 June 2013

Copyright (C) 2013 Xiang Wu et al. This is an open access article distributed under the Creative Commons Attribution License, which permits unrestricted use, distribution, and reproduction in any medium, provided the original work is properly cited.

In recent years, one-dimensional (1D) semiconductor metal oxide nanostructures, such as nanowires, nanorods, and nanotubes, have been the intensive research focus for their potential applications in optical, electrical, optoelectronic, photocatalytic, and hydrophilic/hydrophobic fields. To improve the output efficiency of the nanodevices in various fields, assembling nanostructured building blocks into two-dimensional (2D) or three-dimensional (3D) hierarchical structures is prerequisite.

As guest editors, we are pleased to organize a special issue with a variety of topics within self-assembly of 1D semiconductor nanostructures, as well as the combination of synthesis and application aspects. This is the first special issue on selfassembly of $1 \mathrm{D}$ semiconductor nanostructures that brings together papers concerning recent developments in synthesis and self-assembly of nanocrystals. This special issue consists of a collection of two review articles, seven articles that are contributed from Japan, China, and Israel's scientists.

The review article by $\mathrm{H}$. Zeng's group overviews the fabrication methods of $\mathrm{ZnO}$-based transparent conductive thin films and emphasizes optimum processing parameter conditions and growth mechanism of the as-obtained film on flexible substrate. The review by C. Lai summarizes the synthesis and properties of one-dimensional $\mathrm{TiO}_{2}$ nanomaterials (bare $1 \mathrm{D} \mathrm{TiO}_{2}$ nanomaterial and $1 \mathrm{D}$ hierarchical $\mathrm{TiO}_{2}$ ) and their photovoltaic performance in dye-sensitized solar cells (DSSCs).
The article by $\mathrm{H}$. Zhang et al. studies the effect of alumina dopant on transparency of tetragonal zirconia, and they found that low transparency of the resultant yttria stabilized tetragonal zirconia doped with alumina specimens does not suggest a beneficial effect of alumina dopant even when nanometric grains and high density have been achieved. Finally, they also proposed a possible formation mechanism. X. Wu and coworkers synthesize hierarchical $\mathrm{ZnO}$ nanostructures assembled from nanosheets through a facile hydrothermal route. Their results show that sulfur powder plays a critical role in the morphology of the products. Room temperature photoluminescence property of the hierarchical $\mathrm{ZnO}$ nanostructures shows an ultraviolet emission peak at $385 \mathrm{~nm}$. Scientists from that the Hebrew University demonstrate two alternatives for self-assembled patterning within the nanoscale resolution of optically active semiconductor nanocrystals. The first is substrate selective and uses high-resolution surface patterning to achieve localized self-assembly. The 2nd method uses surface with poly(methyl methacrylate)(PMMA-) resistant patterning adsorption of the nanocrystal with covalent bonds and lift-off. Wang's group reports synthesis of silica-coated $\mathrm{TiO}_{2}$ nanotubes. They discuss the effects of gas-supported process on tubular morphology, crystallinity, and photocatalytic activity. It is found that the sample prepared at hydrothermal treatment and calcination shows perfect open-ended tubular morphology and increased crystallinity. And the photoactivity of the asproduct is proved to be 5 times higher than that of $\mathrm{TiO}_{2}$ 
nanoparticles. X. Wu's group showed large-scale synthesis of $\mathrm{ZnS}$ nanobelts by simply thermal evaporation of $\mathrm{ZnS}$ powder in the presence of $\mathrm{Au}$ catalysts at $1020^{\circ} \mathrm{C}$. Room-temperature photoluminescence measurements showed that the assynthesized $\mathrm{ZnS}$ nanostructures had a strong green emission bands at a wavelength of $427 \mathrm{~nm}$; they attributed it to deep-level emissions induced by defects or impurities. Gao et al. synthesize mesostructured $\mathrm{ZnO}$ with excellent methanol sensing properties by a structure replication procedure through the incipient wetness technique. Their experimental results show that mesostructured $\mathrm{ZnO}$ material shows the higher sensitivity and stability in comparison with the nonporous $\mathrm{ZnO}$ prepared through conventional coprecipitation approach. Finally, X. Wu et al. report formation of flowerlike $\mathrm{Ag}_{2} \mathrm{O} / \mathrm{ZnO}$ heterostructure hybrids by a simple two-step procedure without any surfactants. The as-prepared heterostructure composite showed $\mathrm{Ag}_{2} \mathrm{O}$ nanoparticles coated uniformly on the surface of $\mathrm{ZnO}$ microflowers. They propose a possible growth mechanism of the products based on the experimental results. PL measurements of the as-obtained product reveal a strong UV band-edge emission peak at $383 \mathrm{~nm}$.

\section{Acknowledgments}

The guest editors would like to thank all of the above authors who contributed articles to this special issue for Journal of Nanomaterials. We feel that these contributions provide a cross-section to the current development and future research focuses of self-assembly of $1 \mathrm{D}$ semiconductor nanostructure. We also hope that this collection of articles will inspire researchers and stimulate new ideas in relative research field.

Xiang $W u$

Xijin Xu

Haibo Zeng

Chuanfei Guo 

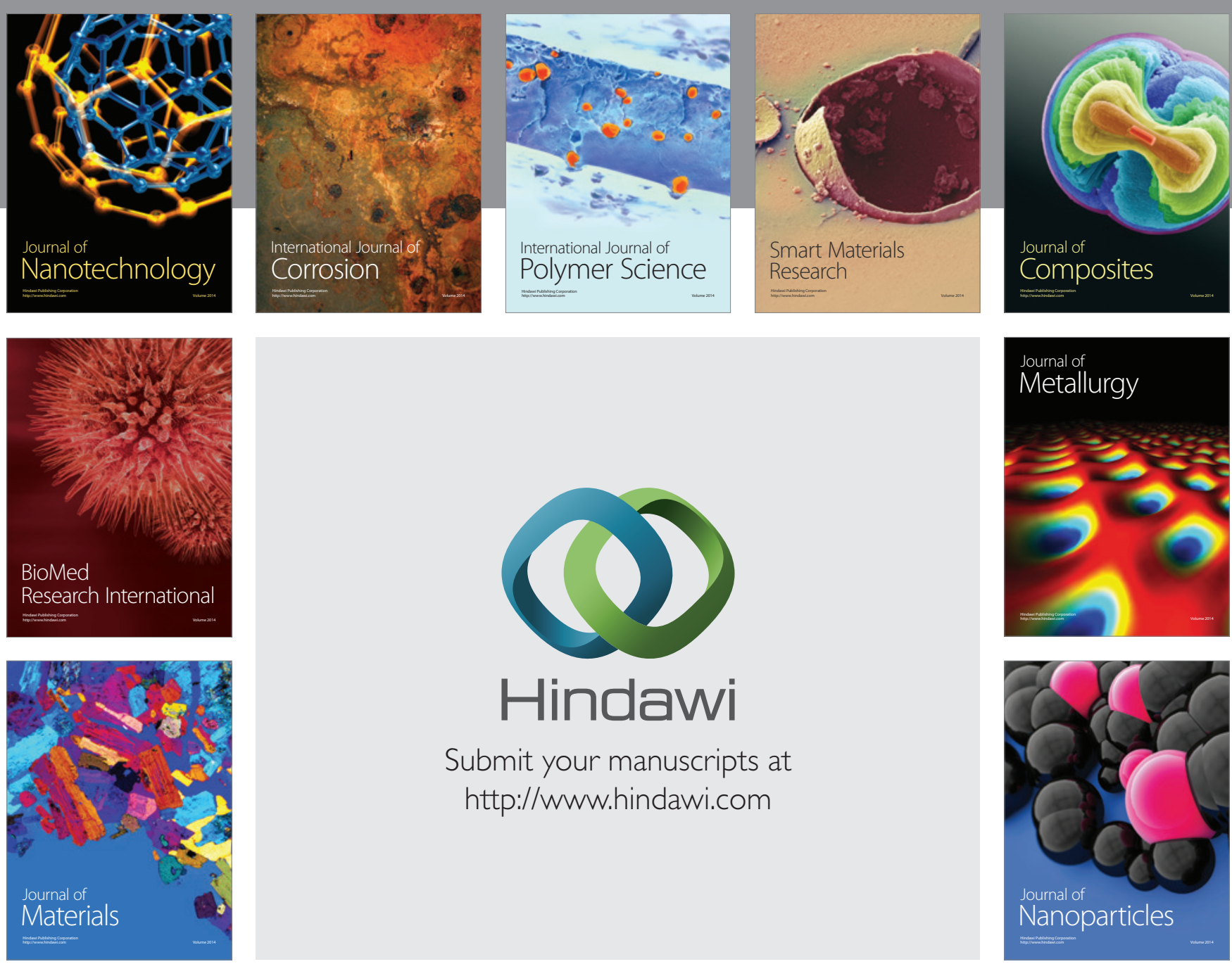

Submit your manuscripts at http://www.hindawi.com
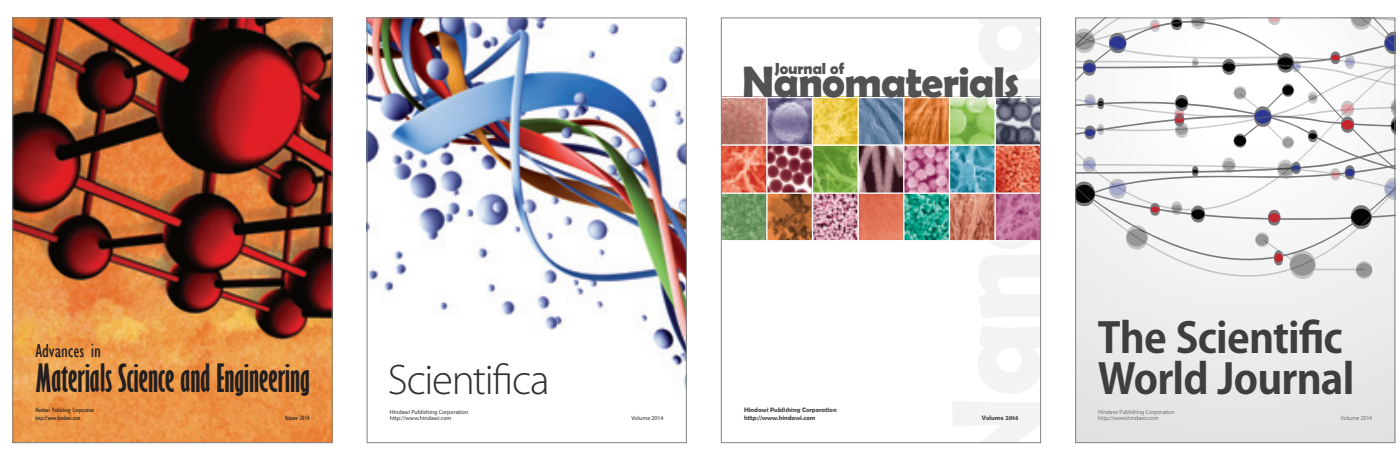

\section{The Scientific World Journal}
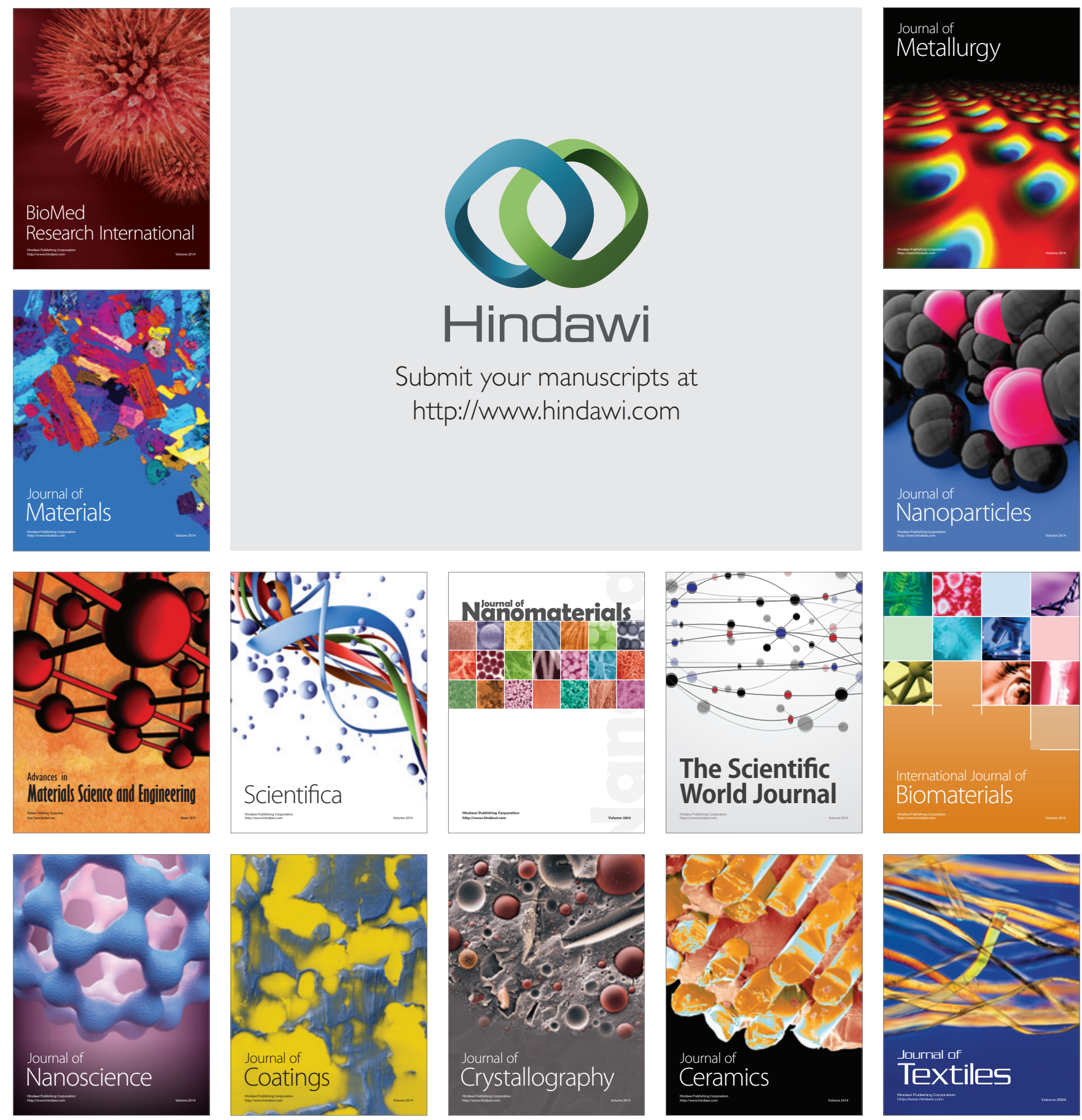